\title{
Hybrid Modulator for power converters in parallel topology
}

\author{
J. Mon, D. González, J. Balcells, J. Gago, P. Bogónez \\ Departament d'Enginyeria Electrònica, Universitat Politècnica de Catalunya. \\ Campus de Terrassa.08222 Terrassa, Spain. juan.mon@upc.edu
}

\begin{abstract}
This paper presents a new mixed modulator that combines interleaving and spread-spectrum techniques in order to achieve the lowest level of conducted EMI generation. This modulator is addressed to power converters in parallel arrangement. The practical details of the hybrid modulator and controller implementation on a FPGA are explained. The characteristics of such modulator in terms of EMI reduction and converter performance are theoretically explored and experimentally validated in a four channel parallel buck converter operating in closed loop.
\end{abstract}

Keywords - Interleaving, frequency modulation, EMI, EMC.

\section{INTRODUCTION}

Switched power converters are operated with constant switching frequency under hard switching regime using Pulse Width Modulation. In a general way, the modulator is the block that generates the switching patterns from the control signal generated by the converter controller. Therefore, it is the block responsible of the main source of EMI. Since switching frequency modulation (SFM) was introduced as EMI reduction technique in switched power converters a lot of works have been published developing this idea [1-11]. At present, it is a well established technique and a worthy alternative to the traditional filtering methods such as the bulky EMI filters or snubber networks in terms of attenuation, cost and simplicity of application. In part due to increase of using programmable logic devices in order to implement digital control systems [11, 13], SFM-modulators are digitally implemented on a field programmable gate array (FPGA) $[10,11]$ or complex programmable logic device (CPLD) [14]. They have been applied to several applications such as home cooking appliance [10] or high performance power supply [14]. However, the application of SFM techniques in distributed or modular power systems made up of several power converters has not been deeply studied yet.

On the other hand, some recent works address the implementation of multiphase digital modulators in order to apply interleaving in parallel multiconverter arrangement, [15, 16]. However, the combined application of interleaving and SFM techniques is an open research field.

This paper presents a new mixed modulator that combines interleaving and spread-spectrum techniques specially intended for conducted EMI suppression. It should be mentioned that this mixed modulator could be applied not only to DC/DC converters but to all kind of multiconverters arrangements.
A thorough description of interleaving can be found in [17- 19].

This paper is organized as follows. First of all, Section II presents the theoretical development of the three hybrid modulations that have been considered. Section III describes the practical implementation used to validate theoretical developments. Section IV summarizes main results that validate the proposed approach. Finally, conclusions are outlined in Section V.

\section{HYBRID MODULATOR THEORY}

Spread Spectrum Modulation in single converter consists of modulating the switching period around a central value, $T_{c}$, according to a given modulation profile. In order to combine the interleaving and spread spectrum modulation in multiconverter arrangement there are several possibilities. Fig. 1 illustrates switching patterns of a multichannel hybrid modulator when the periodic modulation profile is used, where $i$ notes each channel $(i=1,2, \ldots, N), \alpha_{i}$ is the delay among switching patterns, $q_{i}(t)$, and $\varepsilon_{k, i}$ is the pulse position delay inside the $\mathrm{k}^{\text {th }}$ switching cycle. Each switching pattern contains $L$ switching cycles (1). Notice that each switching pattern has a period equal to the period of the modulation profile, $T_{m}$

$L=\frac{T_{m}}{T_{c}}$, Lint eger $>0$

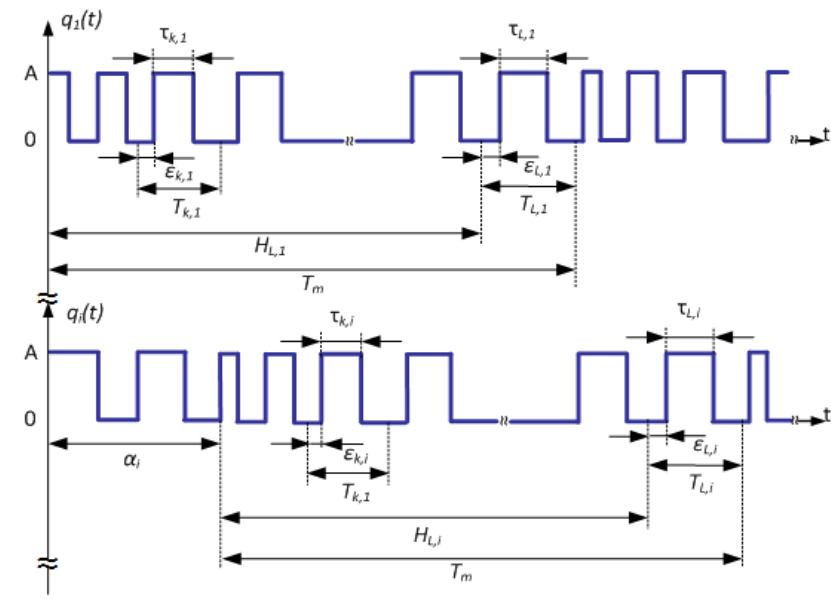

Fig. 1. Generic frequency-modulated switching patterns.

Table I summarizes the modulation characteristics of the three modulation considered in this paper: Constant Delay Tm with switching Frequency Modulation (CDFM-Tm), Constant Delay Tc with switching 
Frequency Modulation (CDFM-Tc) and Variable Delay with switching Frequency Modulation (VDFM). In all cases, a modulation on the switching period is introduced according to a triangular modulation profile. On the other hand, the value of the duty cycle, $D_{c}$, is always established by the controller.

TABLE I

CHARACTERISTICS OF DIFFERENT MODULATIONS

\begin{tabular}{|c|c|c|c|c|}
\hline Modulations & $\varepsilon_{k, i}$ & $T_{k, i}$ & $\tau_{k, i}$ & $\alpha_{i}$ \\
\hline CDFM-Tm & $0 \forall i, k$ & $T_{c}+\Delta T_{k} \forall i$ & $D_{c} \cdot T_{k} \forall i$ & $\frac{T_{m}}{N}(i-1)$ \\
\hline CDFM-Tc & $0 \forall i, k$ & $T_{c}+\Delta T_{k} \forall i$ & $D_{c} \cdot T_{k} \forall i$ & $\frac{T_{c}}{N}(i-1)$ \\
\hline VDFM & $\frac{T_{k, i}}{N}(i-1)$ & $T_{c}+\Delta T_{k} \forall i$ & $D_{c} \cdot T_{k} \forall i$ & $0 \forall i$ \\
\hline
\end{tabular}

In order to evaluate the attenuation provided by the proposed modulations, a time domain description of the switching pattern, $q_{i}(t)$, must be determined.

Considering that a switching pattern has a period equal to the modulation profile period, $T_{m}$, the time domain expression of each of them, $q_{i}(t)$, can be written as (2)

$q_{i}(t)=\sum_{n=-\infty}^{\infty} C_{i, n} e^{\frac{-j 2 \pi n t}{T_{m}}}=C_{i, 0}+2 \sum_{n=1}^{\infty} C_{i, n} e^{\frac{-j 2 \pi n t}{T_{m}}}$

where $C_{i, n}$ are the Fourier coefficients calculated according to (3)

$$
\begin{aligned}
& C_{i, n}=\frac{1}{T_{m}} \int_{0}^{T_{m}} q_{i}(t) e^{\frac{-j 2 \pi n t}{T_{m}}} d t=\frac{A}{j 2 \pi n} e^{\frac{-j 2 \pi n \alpha_{i}}{T_{m}}} \\
& \times \sum_{k=1}^{L} e^{\frac{-j 2 \pi n\left(\varepsilon_{k, i}+H_{k, i}\right)}{T_{m}}}\left(1-e^{\frac{-j 2 \pi n \tau_{k, i}}{T_{m}}}\right)
\end{aligned}
$$

where $A$ is the amplitude of each pattern, $q_{i}(t)$, and $H_{k, i}$ is the starting time of the $\mathrm{k}^{\text {th }}$ switching cycle of each pattern.

Finally, the equivalent source of noise, $s(t)$, in a multiconverter arrangement with $N$ channels is determined by the addition of each particular pattern, $q_{i}(t)$, according to (4).

$$
s(t)=\sum_{i=1}^{N} q_{i}(t)
$$

The modulation parameters shown in Table II were used to compare effects of the proposed modulations. A triangular modulation profile was used in all cases and $A$

\begin{tabular}{|c|c|c|c|c|c|}
\hline \multicolumn{6}{|c|}{ MODULATION PARAMETERS } \\
\hline Modulations & $\begin{array}{c}f_{c} \\
{[\mathrm{kHz}]}\end{array}$ & $\begin{array}{c}\Delta f_{c} \\
{[\mathrm{kHz}]}\end{array}$ & $\begin{array}{c}f_{m} \\
{[\mathrm{kHz}]}\end{array}$ & $\begin{array}{c}D_{c} \\
{[\%]}\end{array}$ & $N$ \\
\hline No modulation & \multirow{4}{*}{300} & 0 & 0 & \multirow{4}{*}{13.5} & \multirow{4}{*}{4} \\
\hline CDFM-Tm & & \multirow{3}{*}{60} & \multirow{3}{*}{10} & & \\
\hline CDFM-Tc & & & & & \\
\hline VDFM & & & & & \\
\hline
\end{tabular}
was set equal to 1 .

TABLE II

\section{A. Constant Delay Tm with switching Frequency} Modulation (CDFM-Tm)

The interleaving effect is obtained by introducing a delay $\alpha_{i}$ among the $N$ switching patterns, which is determined according to the modulation profile, $T_{m}$. Considering the values of Table I, the equivalent source of noise in frequency domain, $S_{C D F M-T m}(w)$, can be expressed by (5)

$$
\begin{aligned}
& S_{C D F M-T m}(w)=F\left\{\sum_{i=1}^{N} q_{i}(t)\right\}=N A D_{c} \partial(w)+\sum_{n=1}^{\infty}\left(\frac{A}{j \pi n}\right. \\
& \left.\times\left[\frac{1-e^{-j 2 \pi n}}{1-e^{\frac{-j 2 m m}{N}}}\right] \sum_{k=1}^{L}\left[e^{\frac{-j 2 \pi H_{k}}{T_{m}}}\left(1-e^{\frac{-j 2 m D_{c} T_{k}}{T_{m}}}\right)\right] \partial\left(w-n w_{m}\right)\right) \\
& =N A D_{c} \partial(w)+N E_{C D F M-T m}(w) \\
& \times \sum_{n=1}^{\infty}\left(\frac{A}{j \pi n} \sum_{k=1}^{L}\left[e^{\frac{-j 2 \pi n H_{k}}{T_{m}}}\left(1-e^{\frac{-j 2 m D_{c} T_{k}}{T_{m}}}\right)\right] \partial\left(w-n w_{m}\right)\right)
\end{aligned}
$$

where $w_{m}$ is corresponds to the modulation profile frequency (6).

$$
w_{m}=2 \pi f_{m}=\frac{2 \pi}{T_{m}}
$$

Notice that the absolute value of $E_{C D F M-T m}(w)$ takes the values given by (7):

$$
\left|E_{C D F M-T m}(w)\right|=\left\{\begin{array}{ll}
0 & n=h N \\
1 & n \neq h N
\end{array} \quad h=0,1,2, \ldots, \infty\right.
$$

Figure 2 illustrates the CDFM-Tm effect. This modulation leads to energy spreading of original harmonics in sidebands. This spectrum contains components at frequencies $n N f_{m}$ only instead of $n f_{m}$ if pure modulation (without interleaving) were applied [4]. For this reason, there are no components at frequencies $f_{c}$, $3 f_{c}$ and $5 f_{c}$ in the resulting spectrum shown in Fig. 2.

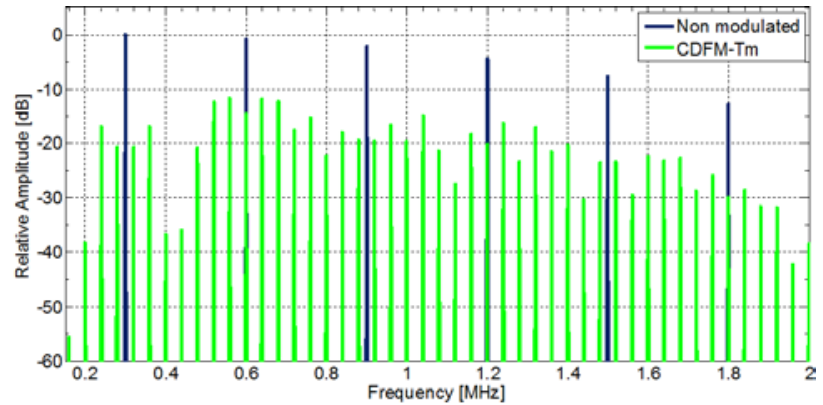

Fig. 2. CDFM-Tm vs. Non modulated.

\section{B. Constant Delay Tc with switching Frequency Modulation (CDFM-TC)}

In this modulation, the delay $\alpha_{i}$ among the switching patterns is calculated from the central value of switching cycle, $T_{c}$. The equivalent source of noise in frequency domain, $S_{C D F M-T c}(w)$, is expressed by (8). 


$$
\begin{aligned}
& S_{C D F M-T c}(w)=F\left\{\sum_{i=1}^{N} q_{i}(t)\right\}=N A D_{c} \partial(w)+\sum_{n=1}^{\infty}\left(\frac{A}{j \pi n}\right. \\
& \times\left[\frac{1-e^{\frac{-j 2 \pi n}{L}}}{1-e^{\frac{-j 2 \pi m}{N L}}} \sum_{k=1}^{L}\left[e^{\frac{-j 2 \pi n H_{k}}{T_{m}}}\left(1-e^{\frac{-j 2 \pi n D_{c} T_{k}}{T_{m}}}\right)\right] \partial\left(w-n w_{m}\right)\right] \\
& =N A D_{c} \partial(w)+N E_{C D F M-T c}(w) \\
& \times \sum_{n=1}^{\infty}\left(\frac{A}{j \pi n} \sum_{k=1}^{L}\left[e^{\frac{-j 2 \pi n H_{k}}{T_{m}}}\left(1-e^{\frac{-j 2 \pi n D_{c} T_{k}}{T_{m}}}\right)\right] \partial\left(w-n w_{m}\right)\right)
\end{aligned}
$$

The term $E_{C D F M-T c}(w)$ provides a energy spread effect that is illustrated in Fig. 3. From Fig. 3 harmonic cancellation is noticed at frequencies $n f_{c}$ except those multiple of $N f_{c}$. In comparison to CDFM-Tm, an additional attenuation is observed for all frequencies except those multiple of $N f_{c}$. Therefore, a better performance than CDFM-Tm is expected. Figure 4 shows the resulting spectrum of CDFM-T . $_{\text {. }}$

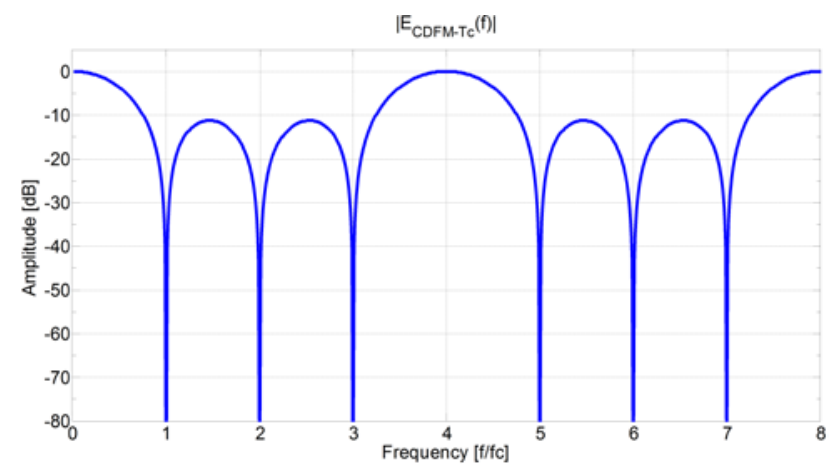

Fig. 3. Envelope of $E_{C D F M-T C}(w)$.

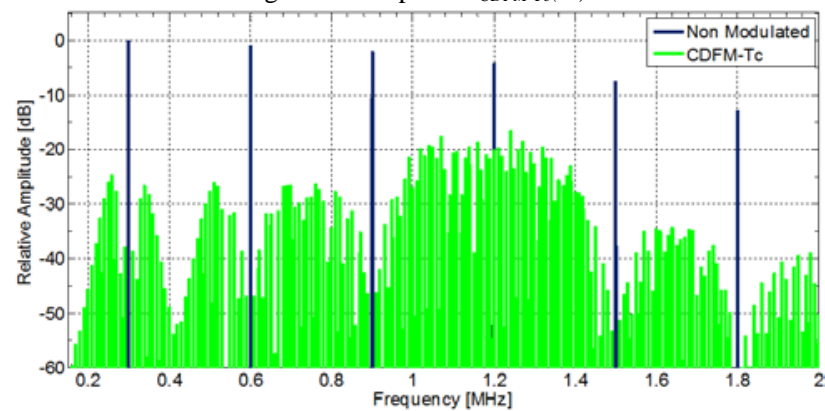

Fig. 4. CDFM-Tc vs. Non modulated.

\section{Variable Delay with switching Frequency Modulation (VDFM)}

In VDFM there is any delay among switching patterns $\left(\alpha_{i}=0\right)$. In this case, a delay on each particular switching cycle, $\varepsilon_{k, i}$, is introduced according to Table I. The equivalent noise pattern in frequency domain, $S_{V D F M}(\omega)$, is expressed by (9).

$$
\begin{aligned}
& S_{V D F M}(w)=F\left\{\sum_{i=1}^{N} q_{i}(t)\right\}=N A D_{c} \partial(w)+\sum_{n=1}^{\infty}\left(\frac{A}{j \pi n}\right. \\
& \times \sum_{k=1}^{L}\left[e^{\frac{-j 2 \pi n H_{k}}{T_{m}}}\left(\frac{1-e^{\frac{-j 2 \pi T_{k}}{T_{m}}}}{1-e^{\frac{-j 2 m T_{k}}{N T_{m}}}}\right)\left(1-e^{\frac{-j 2 \pi D_{c} T_{k}}{T_{m}}}\right) \partial\left(w-n w_{m}\right)\right.
\end{aligned}
$$

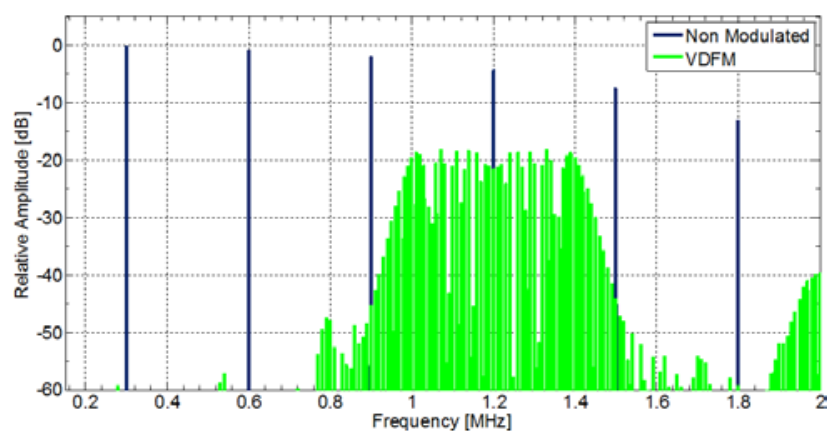

Fig. 5 VDFM vs. Non modulated

Figure 5 illustrates the VDFM effect. The resulting spectrum contains only harmonics of $N f_{c}$, which energy is spread in sidebands. Separation between sidebands is $\mathrm{fm}$. VDFM provides the best results in terms of attenuation. The resulting spectrum of VDFM is equivalent to a single converter operated at $N f_{c}$ with spread spectrum frequency modulation.

\section{HYBRID MODULATOR IMPLEMENTATION}

The hybrid modulator and the digital control loop were implemented on a Spartan-3 FPGA board from Xilinx. Figure 6 shows the block diagram of an n-channels hybrid modulator.

The input signal of the hybrid modulator is the numerical value of the duty cycle, $d$, that comes from the converter controller module. Output signals PWMi goes to the driver of each switching device. This mixed modulator can generate the three above mentioned modulations presented in Section II.

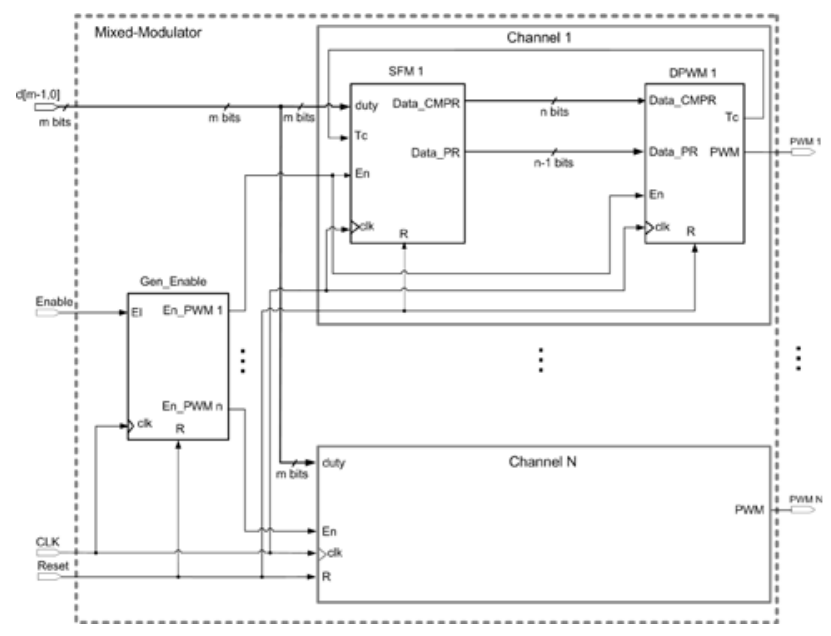

Fig. 6. Block diagram of Hybrid-Modulator.

Each particular channel consists of two modules, Switching Frequency Modulator, (SFMi), and Digital Pulse Width Modulator, (DPWMi). This structure is repeated to obtain a multichannel structure. The module SFM generates the modulation on the switching period and the duty cycle. From these values, module DPWM finally generates the pulse trains for the drivers of the switching devices. The module Gen_Enable manages all the channels of the mixed modulator by generating the delay among the switching pattern of each channel and their enable signals as well. 


\section{A. Gen_Enable}

The basic structure in order to apply a shift delay, $\alpha_{i}$, among $P W M i$ signals is based in a shift register $[18,19]$. However, this approach presents two drawbacks. First, all $P W M i$ signals have the same switching period. On the other hand, this structure does not allow to introduce delays in the pulse position in each switching cycle, $\varepsilon_{k, i}$.

Figure 7 shows the proposed structure in order to apply a shift delay, $\alpha_{i}$. It is based on the counter-comparator architecture. These delays are obtained by introducing variable shift delays among the enable signals for each channel, En_PWMi. This implementation allows having different switching periods for each particular channel.

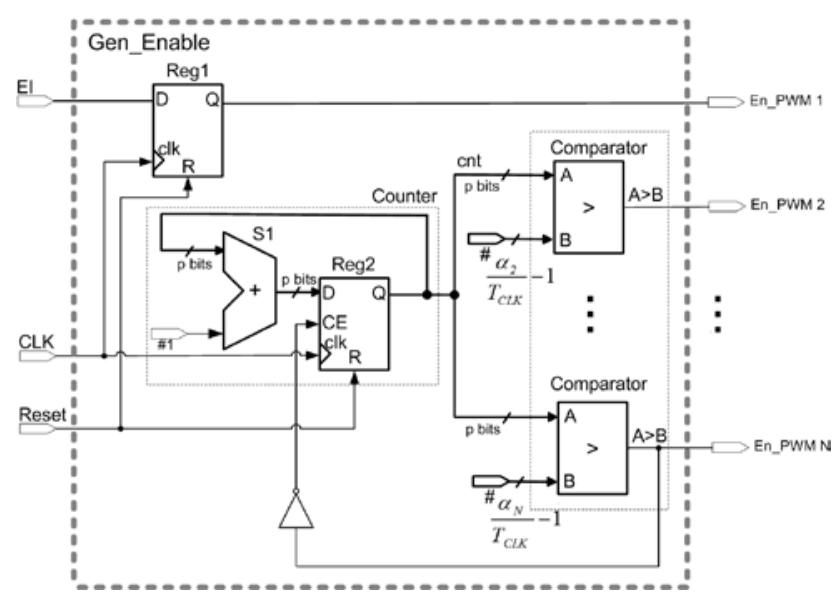

Fig. 7. Block diagram of Gen_Enable.

\section{B. SFM}

SFM module, which block diagram is shown in Fig. 8, is intended to modulate the instantaneous switching period, $T_{k, i}$, of each switching pattern, as it is

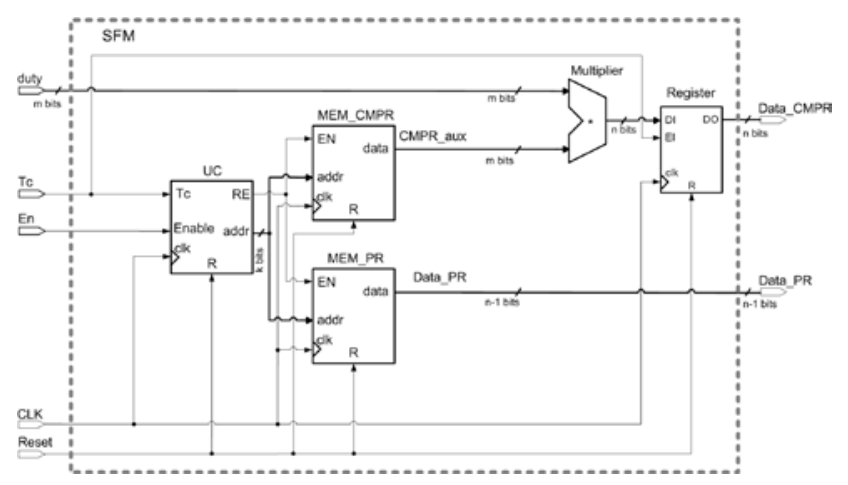

Fig. 8. Block diagram of SFM.

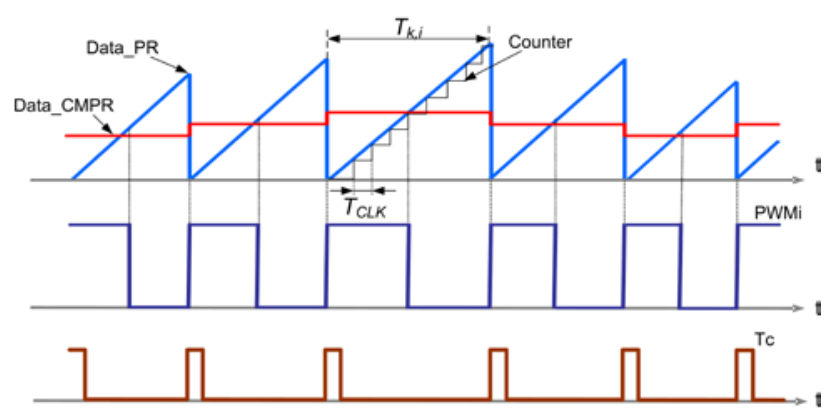

Fig. 9. Spread Spectrum PWM. required by the modulations presented in Section II. This has been done by varying the amplitude of a sawtooth signal, as it illustrated in Fig. 9 where the output signal of the module are shown.

The value of data_CMPR adjusts the duty cycle and Data_PR adjusts the instantaneous switching periods, $T_{k, i}$. The $T_{c}$ signal is used to synchronize the update of Data_CMPR and Data_PR signals at the beginning of each switching cycle. Notice that Data_CMPR is scaled in each switching cycle in order to keep constant the ratio between its value and the varying Data_PR. This results in a correct generation of the duty cycle value that is set by the converter controller on each switching cycle.

There are two memory blocks, MEM_CMPR and MEM_PR, that contains Data_CMPR scaling values and $T_{k, i}$ values, respectively. The value required for Data_CMPR scaling for the current switching period is contained in CMPR_aux signal. Their values are calculated off-line according to (10) and stored in the MEM_CMPR memory block. Regarding MEM_PR memory block, it contains the number of clock cycles required to generate each instantaneous switching periods, $T_{k, i}$. They are calculated off-line according to (11).

$$
\begin{aligned}
& \text { CMPR_aux }=\frac{T_{k, i}}{\min \left\{T_{k, i}\right\}}, k=1,2, \ldots, L \\
& \text { Data_PR }=\frac{T_{k, i}}{T_{c l k}}, k=1,2, \ldots, L
\end{aligned}
$$

The block Control Unit (CU) is a finite state machine that generates all control signals necessary for accessing MEM_CMPR and MEM_PR memory blocks.

In the particular case of modulation VDFM, only the initial pulse position delay for each channel, $\varepsilon_{1, i}$, is introduced by the Gen_Enable module as a delay between PWM patterns. However, the following pulse position delays are introduced by the SFMi modules by modifying the instantaneous switching period of each channel, according to (12). This approach is illustrated by Fig. 10.

$$
\begin{aligned}
& T_{k, i}^{\prime}=T_{k, 1}+\varepsilon_{k+1, i}-\varepsilon_{k, i}=T_{k, 1}+\frac{T_{k+1, i}}{N}(i-1) \\
& -\frac{T_{k, i}}{N}(i-1), k=1,2, \ldots, L-1 \\
& T_{L, i}^{\prime}=T_{L, i}+\varepsilon_{1, i}-\varepsilon_{L, i}= \\
& =T_{L, i}+\frac{T_{1, i}}{N}(i-1)-\frac{T_{L, i}}{N}(i-1), k=L
\end{aligned}
$$

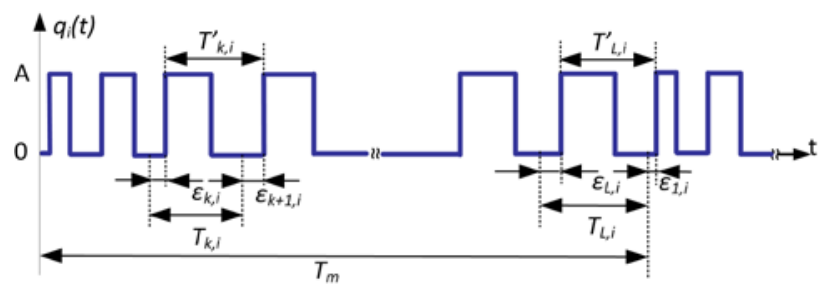

Fig. 10. PWM signals used by VDFM. 


\section{EXPERIMENTAL RESUlTS}

The hybrid modulator described in Section III has been validated in a four channel parallel buck converter. Figure 11 shows the block diagram of the experimental plant. The operating conditions of multichannel buck converter are summarized in Table III.

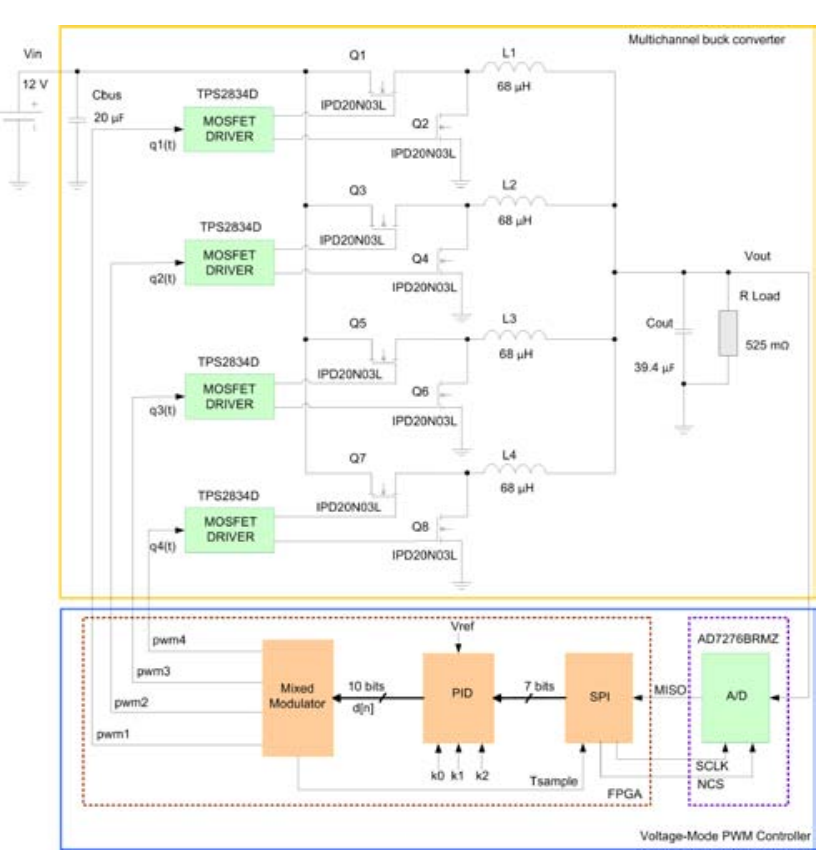

Fig. 11. Block diagram of multichannel buck converter.

TABLE III

MAIN FEATURES OF MULTICHANNEL BUCK CONVERTER

\begin{tabular}{|c|c|c|c|}
\hline Input Voltage & Output Voltage & Power & Switching Frequency \\
\hline $12 \mathrm{~V}_{\mathrm{DC}}$ & $1.5 \mathrm{~V}_{\mathrm{DC}}$ & $4 \mathrm{~W}$ & $300 \mathrm{kHz}$ \\
\hline
\end{tabular}

The digital PID compensator have been implemented according to (13), where $d[n]$ is the duty cycle, $e[n]$ is the error signal and $k 0, k 1$ and $k 2$ are the discrete compensator coefficients.

$$
d[n]=d[n-1]+k_{0} e[n]+k_{1} e[n-1]+k_{2} e[n-2]
$$

The parameters of the voltage PWM controller are summarized in Table IV. The value of $T_{\text {sample }}$ is recalculated on each switching cycle of channel 1 . In our application its values range from $2.77 \mu$ s up to $4.16 \mu \mathrm{s}$.

TABLE IV

PARAMETERS OF THE VOLTAGE PWM CONTROLLER

\begin{tabular}{|c|c|}
\hline K0 & 0.1094 \\
\hline K1 & -0.0938 \\
\hline K2 & 0.0156 \\
\hline
\end{tabular}

In order to evaluate the modulation schemes presented in Section II, the conducted disturbances up to $30 \mathrm{MHz}$ and output voltage ripple are presented.

Measurements of conducted disturbances were carried out using a compliant Line Impedance Stabilization Network (LISN) as show in Fig. 12.

The experimental results have been obtained for a central switching frequency $f_{c}=300 \mathrm{kHz}$, a triangular modulation profile, $V_{m}(t)$, with a modulation frequency $f_{m}=10 \mathrm{kHz}$ and a maximum frequency deviation $\Delta f_{c}=60 \mathrm{kHz}$. The $f_{m}$ parameter has been chosen considering the Resolution Bandwidth (RBW) of the EMI receptor [4].

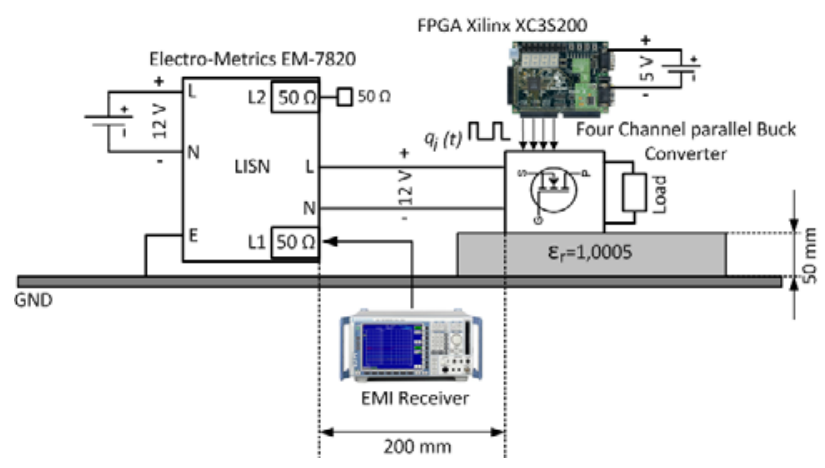

(a)

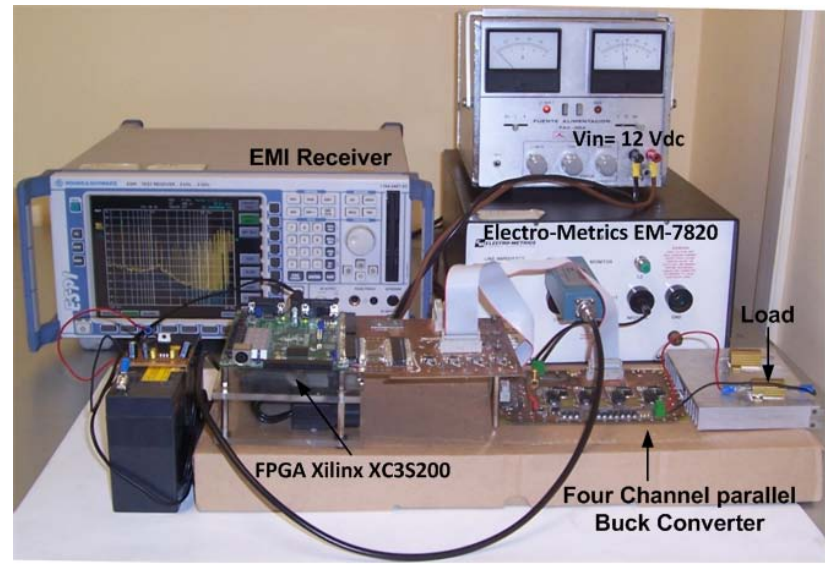

(b)

Fig. 12 EMI measurement set-up. (a) Block diagram. (b) Full view set-up.

Figure 13 shows the spectrum obtained when the converters were operated at constant frequency.

Figures 14, 15 and 16 show the spectrum corresponding to the three proposed modulations.

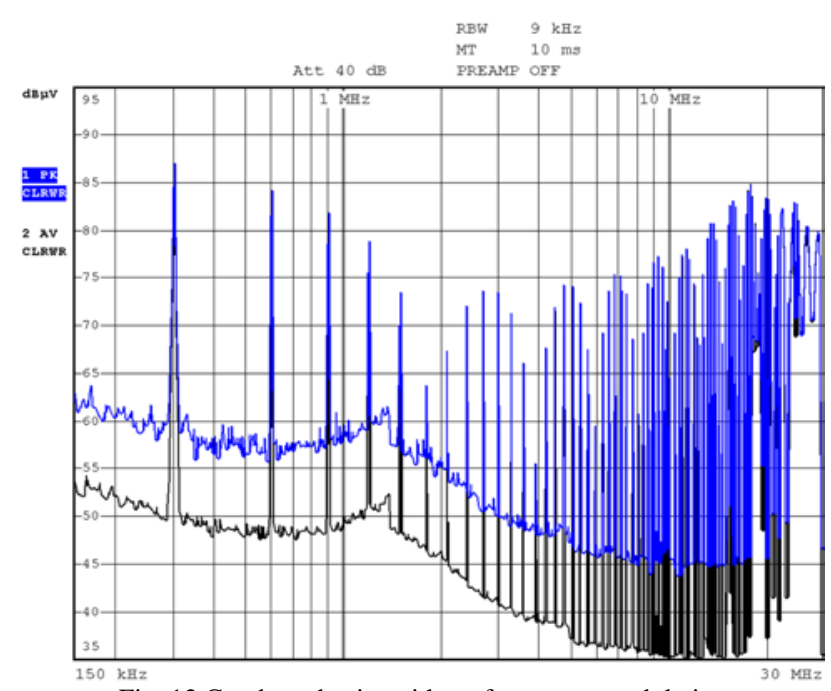

Fig. 13 Conducted noise without frequency modulation. 


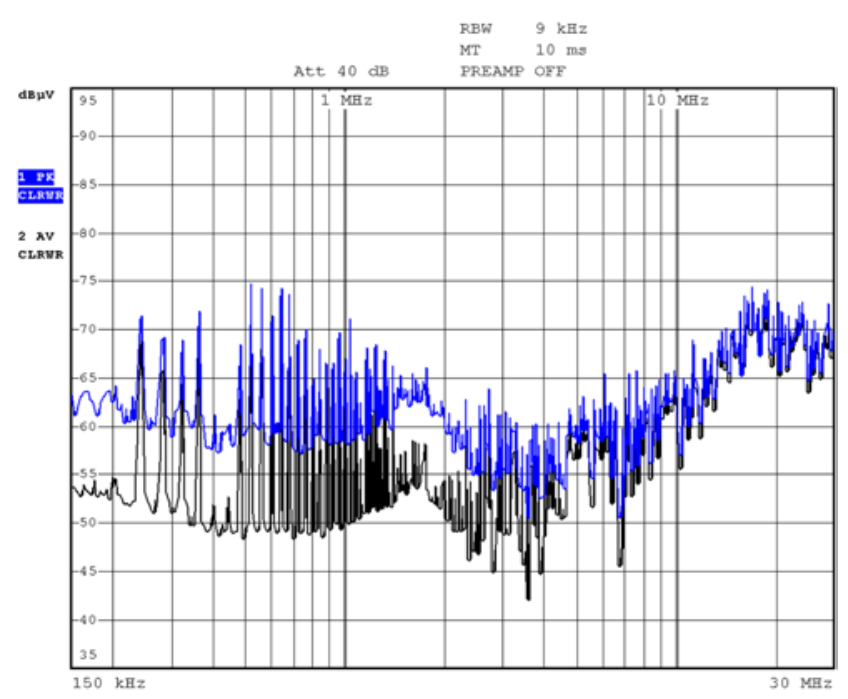

Fig. 14 Conducted noise of CDFM-Tm.

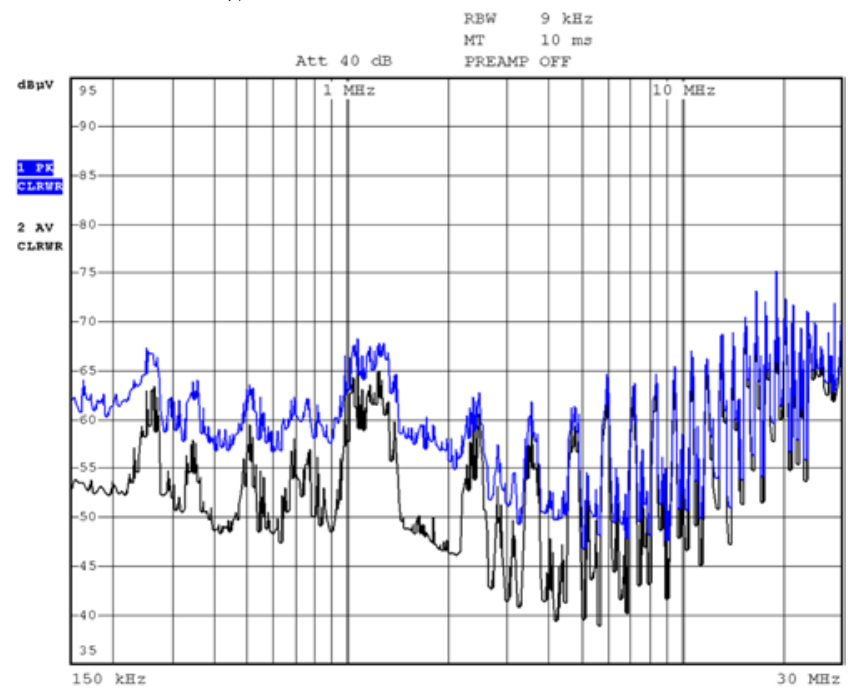

Fig. 15 Conducted noise of CDFM-Tc.

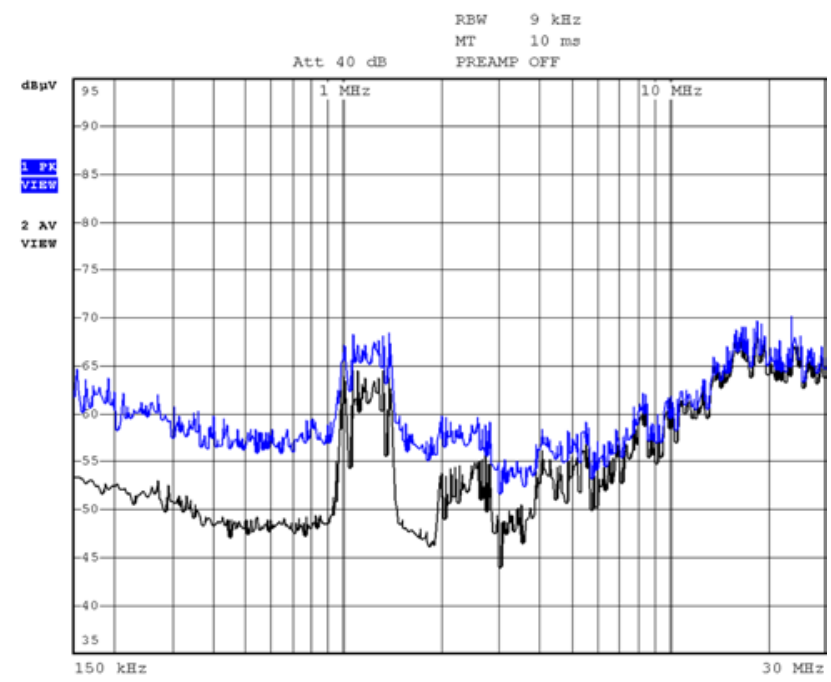

Fig. 16 Conducted noise of VDFM.

As it has been demonstrated in Section II, the CDFM-Tc and VDFM provide the best attenuation. The harmonic cancelation effect of CDFM-Tc that is predicted in Fig. 3 is clearly noticeable in Fig. 15. The best attenuation is given by VDFM. In the low frequency range (up to the 4th harmonic) the cancelation effect is clearly noticed. For higher frequencies, the combination of cancelation and energy spread effects provides an attenuation of almost $15 \mathrm{~dB}$ in all frequency range.

Techniques based on spread spectrum can lead to undesired side effects on the converter performance. It has been demonstrated that power efficiency is not clearly affected [6]. The most noticeable effect will appear as an increase of the output voltage ripple [9]. The output voltage ripple without modulation neither interleaving is $18.4 \mathrm{mV}$.

Figures 17 and 18 compare the output voltage ripple in all cases considered. The modulation frequency, $f_{m}$, is clearly reflected in the output voltage when CDFM-Tc or VDFM are applied. This effect is less noticeable in CDFM-Tm due to the delay introduced among switching patterns.

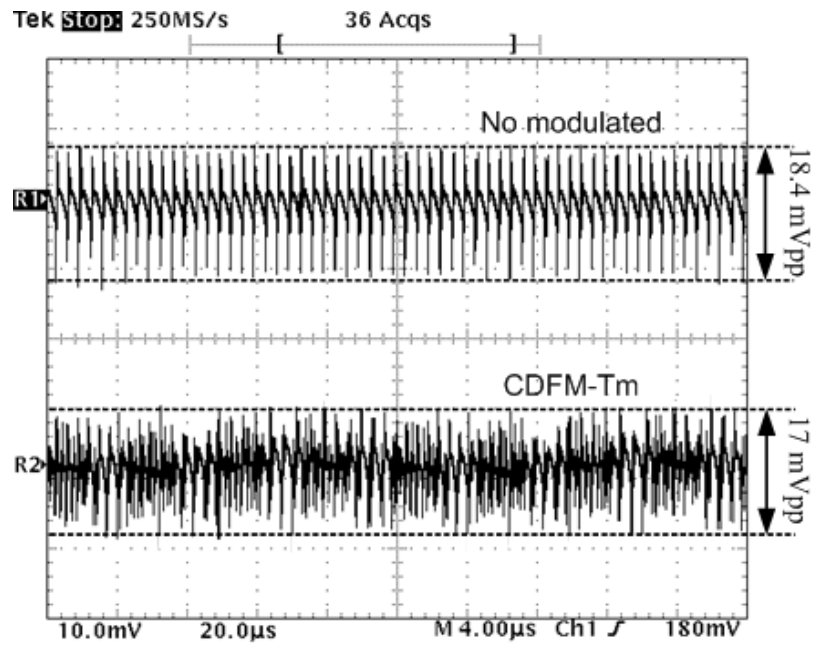

Fig. 17 Output voltage ripple comparison: No modulation and CDFM-Tm (Upper trace: no modulated ; lower trace: CDFM-Tm).

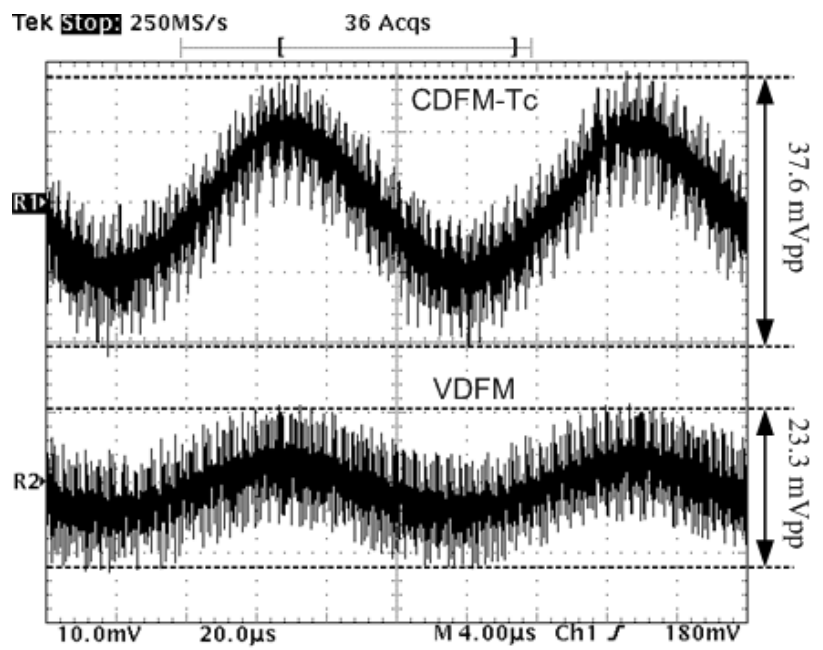

Fig. 18 Output voltage ripple comparison: CDFM-Tc and VDFM (Upper trace: CDFM-Tc ; lower trace: VDFM).

\section{CONCLUSION}

In this paper a hybrid modulator intended to suppress the source of noise inherent to the operation of switched 
power converters is presented. This hybrid modulator implements three combinations of interleaving and spread spectrum. The attenuation provided by such modulators were theoretically explored and practically validated in a four channels buck converter in parallel arrangement and operated in closed loop. EMI attenuation obtained with these techniques was measured on the full bandwidth of conducted disturbances. As a reference to evaluate attenuation, converters were operated at constant frequency and without interleaving. Special attention has been paid to the influence of such techniques on the output voltage ripple of the converter. The VDFM is the technique that provides the best trade-off between attenuation and converter performance degradation in terms of output voltage ripple. CDFM-Tc shows similar attenuation but with worse results in terms of output voltage ripple.

It has been demonstrated that hybrid modulator is a viable and worthy alternative or complementary to conventional passive EMI filters for conducted disturbances attenuation purposes. It should be mentioned that the implementation of these techniques does not require additional components.

\section{ACKNOWLEDGEMENT}

This research is supported by the Ministerio de Ciencia e Innovación in the frame of the project TEC2011-25076.

\section{REFERENCES}

[1] F. Lin and D. Y. Chen, "Reduction of Power Supply EMI Emission bySwitching Frequency Modulation,” IEEE Trans. Power Electron., vol. 9, no. 1, pp. 132-137, January 1994.

[2] A. M. Stankovic, G. E. Verghese, and D. J. Perreault, “Analysis and Synthesis of Randomized Modulation Schemes for Power Converters," IEEE Trans. Power Electron., vol. 10, no. 6, pp. 680-693, November 1995.

[3] K. K. Tse, H. S.-H. Chung, S. Y. Ron Hui, and H. C. So, “A Comparative Study of Carrier-Frequency Modulation Techniques for Conducted EMI Suppression in PWM Converters,” IEEE Trans. Ind. Electron., vol. 49, no. 3, pp. 618-627, June 2002.

[4] J. Balcells, A. Santolaria, A. Orlandi, D. Gonzalez, and J. Gago, "EMI Reduction in Switched Power Converters Using Frequency Modulation Techniques,” IEEE Trans. Electromagn. Compat., vol. 47, no. 3, pp.569-576, August 2005.

[5] S. Johnson and R. Zane, "Custom Spectral Shaping for EMI Reduction in High-Frequency Inverters and Ballasts," IEEE Trans. Power Electron., vol. 20, no. 6, pp. 1499-1505, Nov. 2005.

[6] D. Gonzalez, J. Balcells, A. Santolaria, J.-C. Le Bunetel, J. Gago, D. Magnon, and S. Brehaut, "Conducted EMI Reduction in Power Converters by Means of Periodic Switching
Frequency Modulation,” IEEE Trans. Power Electron., vol. 22, no. 6, pp. 2271-2281, November 2007.

[7] S. Kaboli, J. Mahdavi, and A. Agah, "Application of Random PWM Technique for Reducing the Conducted Electromagnetic Emissions in Active Filters,” IEEE Trans. Ind. Electron., vol. 54, no. 4, pp. 2333-2343, August 2007.

[8] D. Gonzalez, J. T. Bialasiewicz, J. Balcells, and J. Gago, "Wavelet-Based Performance Evaluation of Power Converters Operating With Modulated Switching Frequency,” IEEE Trans. Ind. Electron., vol. 55, no. 8, pp.3167-3176, August 2008.

[9] A. Santolaria, "Effects of Switching Frequency Modulation on the Power Converter's Output Voltage,” IEEE Trans. Ind. Electron., vol. 56, no. 7, pp. 2729-2737, July 2009.

[10] L. A. Barragan, D. Navarro, J. Acero, I. Urriza, and J. M. Burdio, "FPGA Implementation of a Switching Frequency Modulation Circuit for EMI Reduction in Resonant Inverters for Induction Heating Appli- ances,” IEEE Trans. Ind. Electron., vol. 55, no. 1, pp. 11-20, January 2008.

[11] G. Dousoky, M. Shoyama, and T. Ninomiya, "FPGA-Based Spread-Spectrum Schemes for Conducted-Noise Mitigation in DC-DC Power Converters: Design, Implementation, and Experimental Investigation,” IEEE Trans. Ind. Electron., vol. 58, no. 2, pp. 429-435, February 2011.

[12] E. Monmasson, L. Idkhajine, M. Cirstea, I. Bahri, A. Tisan, and M. Naouar, "FPGAs in Industrial Control Applications," IEEE Trans. Ind. Informatics, vol. 7, no. 2, pp. 224 -243, May 2011.

[13] E. Monmasson, L. Idkhajine, and M. Naouar, "FPGA-based Controllers,” IEEE Industrial Electronics Magazine, vol. 5, no. 1 , pp. 14-26, March 2011.

[14] O. Trescases, G. Wei, A. Prodic, and W. Tung, “An EMI Reduction Technique for Digitally Controlled SMPS," IEEE Trans. Power Electron., vol. 22, no. 4, pp. 1560-1565, July 2007.

[15] R. Foley, R. Kavanagh, W. Marnane, and M. Egan, "Multiphase Digital Pulsewidth Modulator," IEEE Trans. Power Electron. vol. 21, no. 3, pp.842-846, May 2006.

[16] M. Scharrer, M. Halton, T. Scanlan, and K. Rinne, "FPGA-Based Multi- Phase Digital Pulse Width Modulator with Dual-Edge modulation," in Applied Power Electronics Conference and Exposition (APEC), 2010 Twenty-Fifth Annual IEEE, February 2010, pp. $1075-1080$.

[17] C. Chang and M. A. Knights, "Interleaving Technique in Distributed Power Conversion Systems," IEEE Trans. Circuits Syst. I, vol. 42, no. 5, pp. 245-251, May 1995.

[18] S. Ozeri, D. Shmilovitz, S. Singer, and L. Martinez-Salamero, "The Mathematical Foundation of Distributed Interleaved Systems,” IEEE Trans. Circuits Syst. I, vol. 54, no. 3, pp. 610 619, March 2007.

[19] P. Zumel, O. Garcia, J. A. Oliver, and J. A. Cobos, "DifferentialMode EMI Reduction in a Multiphase DCM Flyback Converter," IEEE Trans. Power Electron., vol. 24, no. 8, pp. 2013-2020, August 2009.

[19] A. de Castro, G. Glez-de Rivera, O. Garcia, P. Zumel, and T. Riesgo,"Comparison of Phase-shifters for Multiphase Power Converters,” IETE Journal of Research, vol. 57, no. 1, pp. 42-48, March 2011. 